\title{
Inexpensive microcomputer networking for laboratory research
}

\author{
JOSEPH D. ALLEN \\ University of Georgia, Athens, Georgia \\ and \\ LAWRENCE CRAWFORD \\ Temple University, Philadelphia, Pennsylvania
}

\begin{abstract}
Described is a low-cost laboratory network that has allowed us to conduct "on-line" animal research for the past 2 years. A Commodore Model 4032 microcomputer is used to download machine language programs to a network of SYM-1 single-board computers, which in turn each drive several operant stations. Data collected by the SYM-1s are then uploaded to the CBM 4032 after the session, and are then processed and stored on disk.
\end{abstract}

\section{THE PET/CBM AS A STAND-ALONE LABORATORY CONTROLLER}

Since 1980, we have been using models PET 4032 and CBM (Commodore Business Machines) 8032 to serve as on-line controllers for experiments using various clusters of pigeon and rat operant chambers. They have been quite adequate as long as each computer has not serviced more than one or two experimental chambers at a time. The PET/CBM is not a multitasking computer, and with its high overhead of servicing system interrupts (keyboard, screen, memory refresh, etc.), it does not handle external interrupts very reliably. Thus, less efficient polling routines written in either BASIC or machine language must be used to collect data from a few peripheral stations. The PET/CBM also has limited I/O port usability. Although the PET/CBM is equipped with two I/O ports, each containing two 8-bit registers, one entire port, the IEEE port, is best dedicated to servicing a peripheral disk drive and printer. The other, the user port, dedicates one 8-bit register and two control lines to handshaking operations with the IEEE bus, leaving only one unallocated I/O register and two control lines to interface with userdesignated stations. At present, Commodore's PET and new C-64 are both relatively inexpensive (PET: $\$ 500$; C-64: $\$ 200$ ), so one could realistically consider purchasing enough of them to allocate one controller per several stations.

\section{THE PET/SYM-1 LABORATORY NETWORK}

In 1983, when PET/CBMs were still relatively expen-

Joseph D. Allen is with the Department of Psychology, University of Georgia, Athens, GA 30602 . Lawrence Crawford is at 2101 Chestnut St., Philadelphia, PA 19103. sive ( $\$ 1,200-\$ 1,800)$, we decided to expand the number of stations each PET could service by establishing several local laboratory networks, each employing cheaper microcontrollers to directly service the experimental stations. Our choice was the SYM-1 (Synertek Systems), an inexpensive single-board controller, which can be obtained from AB Computers (Colmar, PA) for approximately $\$ 180$.

\section{The SYM-1 as a Dedicated \\ Laboratory Controller}

The SYM-1 has several features that favor its use as a dedicated laboratory controller. First, it comes equipped with two variable-interface adaptors (VIA), the SY6522, and may easily be expanded to three VIAs. Thus, when fully expanded, the SYM-1 provides six 8-bit I/O registers and nine unallocated control lines, yielding a total of 57 I/O lines for the user to interface with peripheral stations. The SYM-1 also provides four empty ROM sockets, which support Synertek's BASIC and assembler packages or, alternatively, support up to $24 \mathrm{~K}$ of user-written programs that may be burned into $2 \mathrm{~K}, 4 \mathrm{~K}$, or $8 \mathrm{~K}$ EPROMs. We routinely use $2 \mathrm{~K}$ EPROMs to house handshaking and data-transfer programs, thus freeing on-board RAM to house our main program as well as store data input from the experimental stations. Unlike the PET/CBM, the SYM-1's internal interrupts are easily disenabled, allowing reliable and dedicated data gathering from peripheral stations using interrupts.

The SYM-1 also has several features that limit its use as a stand-alone controller. On delivery, it contains only $1 \mathrm{~K}$ of "onboard" RAM memory, which, however, may be easily expanded to $4 \mathrm{~K}$ by filling the extra RAM sockets provided with $2114 \mathrm{~s}$. Ultimate RAM expansion to $32 \mathrm{~K}$, however, requires the customized addition of an extra daughter board with concomitant address decoding logic. 
Both the BASIC and assembler packages, which are available as add-ons, are more primitive than similar systems that are either standard or available for the PET/CBM, and working with them is hampered without substituting the small onboard membrane keypad for a more substantive keyboard. Data are output to a six-digit LED display. Although provisions are made on the board for adding either an oscilloscope or monitor, the expense of upgrading the SYM-1 with a suitable keyboard and video display exceeds the cost of available, fully configured microcomputers.

\section{The PET/SYM-1 Network}

Placing the PET/CBM and SYM-1 computers in a network in which clusters of SYM-1s serve as dedicated controllers of our peripheral stations and are in turn slaved to a single PET/CBM accentuates the best features of both computers. Figure 1 displays one currently working system, in which one Commodore 4032 station, with disk drive and printer, coordinates two SYM-1 controllers, which in turn each service two operant boxes. Since the two computers share the same instruction set ( 6502 based), we can use the full-featured MAE assembler/developer package (Eastern Software House) on the PET to develop machine language programs to run on the SYM-1s. At the beginning of the session, the programs are loaded into the PET from the disk drive via the IEEE bus and are downloaded into the SYM-1's memory using the 8-bit A register on the PET's user port. Since the PET's operating system does not implement handshaking routines on its user port, data-transfer programs must be written for both the PET and the SYM-1. Download and upload routines were written to reside at locations \$A000 and \$A100 in an empty ROM slot in the PET/CBM, and comparable routines were written to reside at unused ROM addresses \$D000 and \$D100 on the SYM-1. Both source and object codes for the PET and SYM-1 upload and download boots are available from us upon request. The download boot initializes the PET's port as an output and then requests the SYM's start address (usually \$0200) and the PET's start address and end address as inputs from the keyboard. Then, in conjunction with the SYM's download boot, it handshakes the block of program data from the PET via the user port bus to the SYM's VIA \#2 chip, which is addressed at $\$ A 800$ on the SYM's AA port. Raw data at the end of each session are handshaked from the SYM-1 to the PET/CBM using the pair of upload boots in similar fashion. In the PET, data-handler programs, written in compiled BASIC, perform any desired data crunching before producing hardcopy summaries on the printer and storing the raw data in sequence files on disk using packed binary format.

Whereas the PET and SYM-1s are easily linked via a parallel cable and a pair of edge connectors, interfacing between the SYM-1s and the operant boxes usually requires more sophisticated logic. Our University Electronics Shop has constructed interface modules that allow each SYM-1 to drive the operant box's pellet dis-

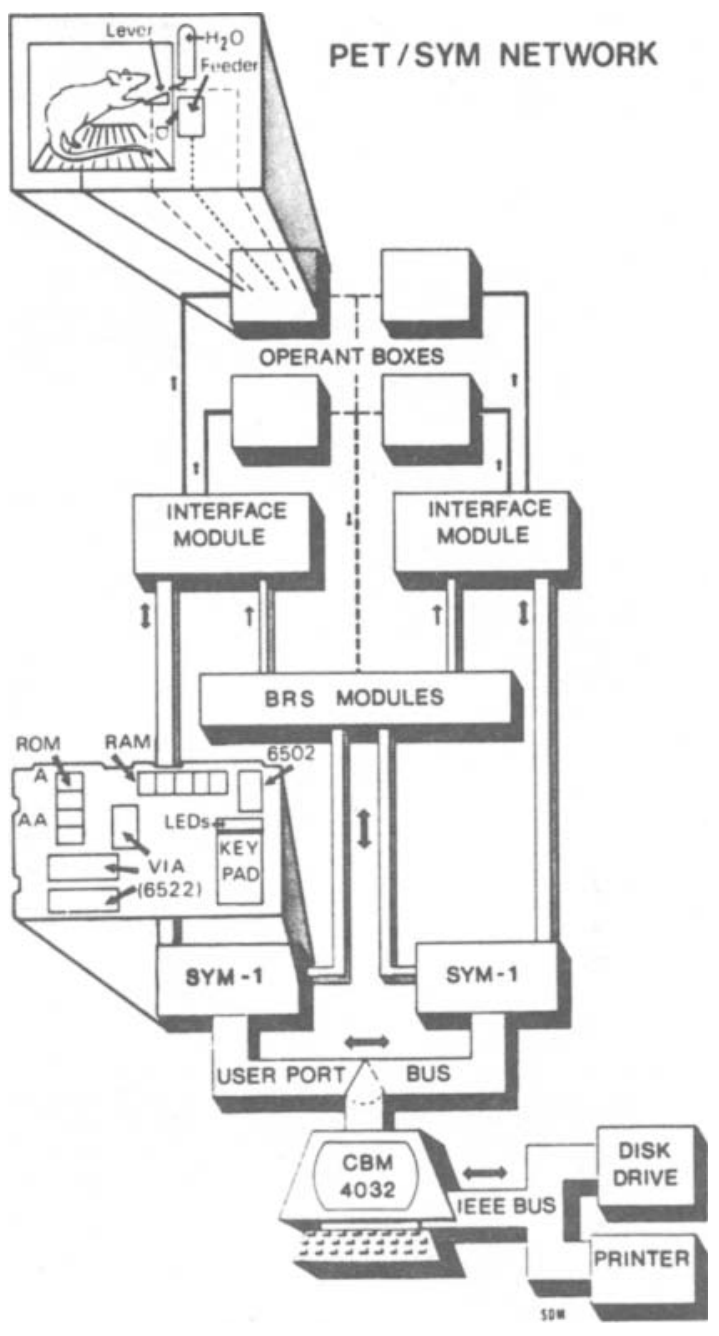

Figure 1. A laboratory network linking a PET/CBM to two SYM-1s, which each service two operant stations. The authors are grateful to Susan D. Meier for designing and rendering this illustration.

pensers and other output devices that require high inductive loads. Leverpresses and tongue licks produced by the rat in the box are in turn conditioned into clean $20-\mathrm{msec}$ signals by the interface. They are then sent out to the SYM-1 via control lines, where they generate interrupts that are then serviced by appropriate handler routines in the SYM. The inputted data are tabulated in memory arrays lying directly above the RAM locations containing the program code. A schematic diagram of the interface is available from us for a $\$ 5.00$ handling charge and a SASE mailer. In our system, BRS/LVE logic modules (any solid-state logic will do) intercede between the SYMs, the interface modules, and the operant boxes. They are used to sense tube contacts (BRS drinkometer) and to time pulse durations to the feeders and liquid dispensers, thus relieving the SYM-1 of a costly timing duty. 
Although minimally configured, the network depicted in Figure 1 may easily be expanded to control an extensive system of work stations. After discounting the 8 data lines and 2 control lines that permit handshaking with the PET, there remain 40 data and 7 control lines on each SYM-1, which may be used in various configurations to service a cluster of peripheral stations. Also, since the SYM-1s operate as autonomous controllers during a session's run, the network may be expanded indefinitely at the next level by adding extra SYM-1s at a cost of $\$ 180$ per unit plus the cost of an interface module. The PET/CBM merely serves to download programs to each unit at the beginning of each session and to collect data from each unit in series at the end of each session. The PET, of course, is freed during the session to perform other tasks, such as data analysis and plotting. 